\title{
Biology of three species of North American Triatominae (Hemiptera: Reduviidae: Triatominae) fed on rabbits
}

\author{
José Alejandro Martínez-Ibarra/ ${ }^{+}$, Ricardo Alejandre-Aguilar*, \\ Edgar Paredes-González**, Miguel Alejandro Martínez-Silva, Miriam Solorio-Cibrián, \\ Benjamín Nogueda-Torres**, Francisco Trujillo-Contreras, Mónica Novelo-López
}

\begin{abstract}
Área de Entomología Médica, Centro Universitario del Sur, Universidad de Guadalajara, A. P. 20, 49000 Ciudad Guzmán, Jalisco, México *Departamento de Ciencias Químico Biológicas y Agropecuarias, Universidad de Sonora, Unidad Norte Caborca, Caborca, Sonora, México ** Becario de COFAA, Escuela Nacional de Ciencias Biológicas, Instituto Politécnico Nacional, Carpio y Plan de Ayala, Colonia Casco de Santo Tomás, México, D. F.
\end{abstract}

Aspects related to hatching, lifetime, number of blood meals for molting, mortality, feeding time, and postfeeding defecation delay were evaluated and compared in each instar of three North American Triatominae: Triatoma gerstaeckeri, Triatoma lecticularia and Triatoma protracta, all of them fed on rabbits. No significant differences $(p>0.05)$ were found among the three species regarding mean hatching rate, which was close to 20 days. Egg-to-adult development times were significantly shorter $(p<0.05)$ for T. lecticularia. Number of blood meals for molting to next instar ranged from one to five for $\mathrm{T}$. protracta, and from one to six for $\mathrm{T}$. gerstaeckeri and $\mathrm{T}$. lecticularia. Mortality rates were higher in younger nymphs of $\mathrm{T}$. lecticularia and $\mathrm{T}$. protracta, while rates in T. gerstaeckeri were higher in fifth-instar nymphs. Mean feeding time was longest in T. gerstaeckeri, followed by $\mathrm{T}$. lecticularia. More than twice the number of T. gerstaeckeri nymphs completed the development process, if compared to the nymphs from the other two species. Defecation delay was less than 10 min for T. lecticularia, T. protracta and the youngest nymphs of $\mathrm{T}$. gerstaeckeri. Results point out that these three species may be important potential vectors of Trypanosoma cruzi for human populations, in areas of Mexico where these species are currently present.

Key words: North America - Triatominae -biology - laboratory conditions

Chagas disease is considered an emerging illness in the United States. Although only a few chronic cases have been detected in that country, a significant number of presumed cases of American trypanosomiasis has been recorded, as well as at least twelve different species of reservoir hosts (Navin et al. 1985, Hanford et al. 2007) and a near number of triatomine species (Galvão et al. 2003). In Mexico, on the other hand, it is believed that Chagas disease represents an important problem (CruzReyes \& Pickeri-López 2006), with foci in some states on the USA border (e.g., Chihuahua, Sonora and Nuevo León) (Paredes et al. 2001, Licón-Trillo 2006, MolinaGarza et al. 2006), and also other probable foci in other border states of Baja California, Coahuila and Tamaulipas. Triatoma gerstaeckeri (Stål) is one of the major species of Triatominae in states on both sides of the USAMexico border, whereas Triatoma lecticularia (Stål) and Triatoma protracta (Uhler) are regionally important on that area. However, the three species stand out due to their distribution, association with human dwellings, and entomological indexes (Zárate \& Zárate 1985, GalavízSilva et al. 1991a, Martínez-Ibarra et al. 1992, Beard et

+Corresponding author: aibarra@cusur.udg.mx Received 25 June 2007

Accepted 27 November 2007 al. 2003, Galvão et al. 2003, Molina-Garza et al. 2006, 2007, Hanford et al. 2007). Studies on the behavior of triatomine species help to widen knowledge about their habits, what results in improving awareness with regard to decisions for vector control. Several studies have been devoted to specific aspects of behavior in $T$. gerstaeckeri and T. lecticularia (Pippin 1970, Jurberg \& Costa 1989, Galavíz-Silva et al. 1991b, Torres-Estrada et al. 1993, 2002, García-Pérez et al. 1997, Seigler \& Lampman 2000). However, life cycles and feeding and defecation behaviors of $T$. gerstaeckeri have been sparsely evaluated. In Pippin (1970), T. gerstaeckeri were fed on laboratory white mice or on adult white rats, and in Galavíz-Silva et al. (1991b), specimens were fed on white mice (1st-2nd instars) and on rabbits (3rd-5th instars and adults). Similarly, only few studies have been dedicated to investigate some of those biological behaviors in T. lecticularia, like one about fasting (Jurberg \& Costa 1989), another study about life cycle, fed on rabbits (Silva et al. 1993), and that devoted to research about defecation patterns on T. lecticularia, fed fortnightly on rabbits (Nogueda-Torres et al. 2000). On the other hand, any study has been carried out on those biological parameters in $T$. protracta.

As part of a series of studies on the biology of Mexican Triatominae, the present study was conducted to examine life cycle, feeding and defecation behavior of one of the major species and two regionally important species in the transmission process of Trypanosoma cruzi to human populations from Mexico and, potentially, from the USA. 


\section{MATERIALS AND METHODS}

The laboratory colonies of $T$. gerstaeckeri and $T$. lecticularia used in this study were initiated in 2005 from specimens collected in Linares, Nuevo León. The colony of $T$. protracta was created in 2005 from specimens originally obtained in Caborca, Sonora. Colonies were maintained at $27 \pm 1^{\circ} \mathrm{C}$, in a relative humidity $(\mathrm{RH})$ of $30 \pm 2 \%$, and in a $12 / 12 \mathrm{~h}$ (light/darkness) regimen, conditions that are characteristic of the areas where these three species usually live. Triatomines were weekly fed on immobilized New Zealand rabbits.

Eggs of each species were grouped by oviposition date in order to initiate a cohort of 200 eggs each per species. After hatching, the first-instar nymphs were placed separately in individual plastic containers $(5.5 \mathrm{~cm}$ diameter x $10.5 \mathrm{~cm}$ height) with a central upright absor-

\section{TABLE I}

Hatching rate and mean incubation period in Triatoma gerstaeckeri, T. lecticularia and T. protracta fed every seven days on rabbits

\begin{tabular}{lcc}
\hline Species & Hatching rate (\%) & $\begin{array}{c}\text { Mean incubation } \\
\text { period in days (range) }\end{array}$ \\
\hline T. gerstaeckeri & 75.0 & $22.4 \pm 8.7(15-28)$ \\
T. lecticularia & 82.5 & $18.9 \pm 7.4(14-24)$ \\
T. protracta & 76.0 & $20.7 \pm 9.4(19-25)$ \\
\hline
\end{tabular}

bent cardboard support. Three days after hatching, the nymphs were individually fed on New Zealand rabbits for $1 \mathrm{~h}$. Subsequent blood meals were provided weekly. Nymphs were observed from the beginning of the feeding process until $1 \mathrm{~h}$ postfeeding in order to record feeding and defecation behavior. The bugs were maintained in a dark incubator at a temperature of $27 \pm 1^{\circ} \mathrm{C}$ and $30 \pm$ $2 \% \mathrm{RH}$, and they were daily checked for ecdysis or death. Among the insects that completed development to adult instar, ten couples from each cohort were placed in separated plastic containers $(5.5 \mathrm{~cm}$ diameter $\mathrm{x} 10.5 \mathrm{~cm}$ height), and maintained as described above to determine oviposition patterns.

Variables showing a normal distribution were compared using Student's t-test or analysis of variance (ANOVA). In the case of ANOVA, post hoc comparisons were made using the Scheffé test. The Wilcoxon nonparametric test was used for variables with abnormal distribution. The chi-square test was used to compare frequencies. Differences were considered significant when $\mathrm{p}<0.05$.

\section{RESULTS}

Egg eclosion rate was over $70 \%$ in all cohorts, with mean incubation periods of about 20 days in all three species. No significant $(p>0.05)$ differences were found among them (Table I). Average time for egg-to-adult development was seven months or more. Significant differ-

TABLE II

Egg-to-adult developmental cycle in Triatoma gerstaeckeri, T. lecticularia and T. protracta fed every seven days on rabbits

\begin{tabular}{lcccccc}
\hline \multicolumn{7}{c}{ Duration in days (range) } \\
\hline Instar & Number & Mean \pm SD & Number & Mean \pm SD & Number & Mean \pm SD \\
\hline Egg-NI & 150 & $22.7 \pm 0.7(19-24)$ & 165 & $18.9 \pm 7.4(16-27)$ & 152 & $19.2 \pm 8.4(17-28)$ \\
NI-NII & 138 & $22.6 \pm 0.6(20-23)$ & 99 & $29.2 \pm 8.8(21-39)$ & 91 & $34.6 \pm 15.5(19-85)$ \\
NII-NIII & 127 & $21.8 \pm 1.1(19-34)$ & 80 & $54.1 \pm 20.6(32-77)$ & 75 & $49.6 \pm 28.1(20-62)$ \\
NIII-NIV & 119 & $41.8 \pm 0.7(35-44)$ & 58 & $58.6 \pm 24.6(31-86)$ & 58 & $54.0 \pm 34.0(15-85)$ \\
NIV-NV & 109 & $59.3 \pm 1.3(33-98)$ & 49 & $44.2 \pm 20.9(23-79)$ & 51 & $64.6 \pm 38.6(28-155)$ \\
NV-AD & 90 & $97.9 \pm 2.8(75-119)$ & 41 & $67.8 \pm 19.5(42-104)$ & 48 & $70.5 \pm 40.9(32-161)$ \\
Total & 90 & $278.6 \pm 6.8^{a}(255-313)$ & 41 & $234.9 \pm 20.6^{a}(201-262)$ & 48 & $269.6 \pm 35.7^{a}(196-331)$ \\
\hline
\end{tabular}

$a: \mathrm{p}<0.05$. NI-NV: 1 st to 5 th instar nymphs.

TABLE III

Mean number of blood meals for molting in Triatoma gerstaeckeri, T. lecticularia and T. protracta fed every seven days on rabbits, under laboratory conditions

\begin{tabular}{|c|c|c|c|c|c|c|}
\hline \multirow[b]{3}{*}{ Instar } & \multicolumn{6}{|c|}{ Number of blood meals (range) } \\
\hline & \multicolumn{2}{|c|}{ T. gerstaeckeri } & \multicolumn{2}{|c|}{ T. lecticularia } & \multicolumn{2}{|c|}{ T. protracta } \\
\hline & Number & Mean $\pm \mathrm{SD}$ & Number & Mean $\pm \mathrm{SD}$ & Number & Mean $\pm \mathrm{SD}$ \\
\hline NI-NII & 150 & $1.4 \pm 0.1(1-3)$ & 165 & $2.4 \pm 0.52(2-5)$ & 152 & $2.8 \pm 1.0(2-5)$ \\
\hline NII-NIII & 138 & $2.2 \pm 0.1(1-3)$ & 99 & $3.4 \pm 1.12(2-5)$ & 91 & $1.8 \pm 0.7(1-5)$ \\
\hline NIII-NIV & 127 & $4.1 \pm 0.1(1-5)$ & 80 & $3.1 \pm 2.71(2-5)$ & 75 & $2.1 \pm 1.0(1-4)$ \\
\hline NIV-NV & 119 & $3.6 \pm 0.2(2-6)$ & 58 & $2.2 \pm 0.69(3-6)$ & 58 & $2.7 \pm 1.3(2-5)$ \\
\hline NV-AD & 109 & $4.7 \pm 1.3(2-6)$ & 49 & $3.2 \pm 1.05(3-6)$ & 51 & $3.7 \pm 1.5(2-5)$ \\
\hline Total & 90 & $13.2 \pm 0.2(11-16)$ & 41 & $14.9 \pm 1.9(14-20)$ & 48 & $12.6 \pm 2.9(10-18)$ \\
\hline
\end{tabular}

NI-NV: 1 st to 5 th instar nymphs. 
ences $(\mathrm{p}<0.05)$ occurred between T. gerstaeckeri and $T$. lecticularia, and between T. protracta and $T$. lecticularia, but not between $T$. gerstaeckeri and $T$. protracta (Table II).

Mean number of blood meals for molting did not differ significantly $(\mathrm{p}>0.05)$ among the three species (Table III). Mortality rates were considerably lower $(\mathrm{p}>0.05)$ for $T$. gerstaeckeri than for the other two species, but no significant $(\mathrm{p}>0.05)$ difference was observed when T. lecticularia and T. protracta were compared (Table IV). At the end of the egg-to-adult development cycle, there was a significantly different $(\mathrm{p}<0.05)$ number of males and females for $T$. gerstaeckeri, while T. lecticularia and T. protracta had a similar number of specimens from each sex (Table V).
Mean feeding time (FT) was significantly longer (p $<0.05$ ) than $10 \mathrm{~min}$ in most instars of T. gerstaeckeri and $T$. lecticularia, but remained close to $10 \mathrm{~min}$ in most instars of $T$. protracta. Older (4th and 5th instars) nymphs and adults of $T$. gerstaeckeri had significantly longer $(p<0.05)$ FTs than most of younger nymphs $(1 \mathrm{st}$ and 3rd instars). On the other hand, FTs did not differ significantly $(\mathrm{p}<0.05)$ among most instars of $T$. lecticularia, while longer FTs were observed in fifthinstar nymphs and females of T. protracta, although no significant differences $(\mathrm{p}<0.05)$ were found $($ Table V).

Postfeeding defecation delay was less than 10 min only in younger nymphs of T. gerstaeckeri, and in all instars of T. lecticularia and T. protracta (Table VI).

TABLE IV

Instar mortality in Triatoma gerstaeckeri, T. lecticularia and T. protracta fed on rabbits, under laboratory conditions

\begin{tabular}{lcccccc}
\hline & \multicolumn{2}{c}{ T. gerstaeckeri } & \multicolumn{2}{c}{ T. lecticularia } & \multicolumn{2}{c}{ T. protracta } \\
\hline Instar & Number & \% mortality & Number & \% mortality & Number & \% mortality \\
\hline NI & 150 & 8.0 & 169 & 41.4 & 152 & 40.1 \\
NII & 138 & 6.0 & 99 & 11.2 & 91 & 10.5 \\
NIII & 127 & 5.3 & 80 & 13.0 & 75 & 11.2 \\
NIV & 119 & 6.7 & 58 & 5.3 & 58 & 4.6 \\
NV & 109 & 12.7 & 49 & 4.7 & 51 & 2.0 \\
Total & 90 & $(38.7)^{a}$ & 41 & $(75.6)^{a}$ & 48 & $(68.4)^{a}$ \\
\hline
\end{tabular}

$a: \mathrm{p}<0.05 . \mathrm{NI}-\mathrm{NV}: 1$ st to 5 th instar nymphs.

TABLE V

Feeding times in minutes for Triatoma gerstaeckeri, T. lecticularia and T. protracta fed on rabbits, under laboratory conditions

\begin{tabular}{|c|c|c|c|c|c|c|}
\hline \multirow[b]{2}{*}{ Instar } & \multicolumn{2}{|c|}{ T. gerstaeckeri } & \multicolumn{2}{|c|}{ T. lecticularia } & \multicolumn{2}{|c|}{ T. protracta } \\
\hline & Number & Minutes (range) & Number & Minutes (range) & Number & Minutes (range) \\
\hline NI & 150 & $18.9 \pm 1.1^{a}(7-22)$ & 99 & $19.7 \pm 8.1(4-26)$ & 152 & $9.1 \pm 5.4(4-27)$ \\
\hline NII & 138 & $29.8 \pm 0.9^{a}(4-30)$ & 80 & $19.1 \pm 7.2(3-26)$ & 91 & $9.4 \pm 3.9(3-26)$ \\
\hline NIII & 127 & $21.8 \pm 1.2^{a}(7-24)$ & 58 & $17.4 \pm 7.7(4-26)$ & 75 & $11.3 \pm 6.7(4-27)$ \\
\hline NIV & 119 & $38.8 \pm 2.1^{a}(5-23)$ & 49 & $20.3 \pm 8.7(3-25)$ & 58 & $10.4 \pm 6.3(5-28)$ \\
\hline NV & 109 & $37.9 \pm 1.5^{a}(5-23)$ & 41 & $24.1 \pm 7.5(5-26)$ & 51 & $17.5 \pm 8.9(4-29)$ \\
\hline Females & 53 & $37.5 \pm 1.7^{a}(5-26)$ & 22 & $21.2 \pm 7.7(4-29)$ & 25 & $15.5 \pm 5.7(5-30)$ \\
\hline Males & 37 & $34.2 \pm 1.6^{a}(6-25)$ & 19 & $20.7 \pm 7.5(3-29)$ & 23 & $12.0 \pm 4.24(4-27)$ \\
\hline
\end{tabular}

$a: \mathrm{p}<0.05$. NI-NV: 1 st to 5 th instar nymphs.

TABLE VI

Defecation times in minutes for Triatoma gerstaeckeri, T. lecticularia and T. protracta fed on rabbits, under laboratory conditions

\begin{tabular}{|c|c|c|c|c|c|c|}
\hline \multirow[b]{2}{*}{ Instar } & \multicolumn{2}{|c|}{ T. gerstaeckeri } & \multicolumn{2}{|c|}{ T. lecticularia } & \multicolumn{2}{|c|}{ T. protracta } \\
\hline & Number & Minutes (range) & Number & Minutes (range) & Number & Minutes (range) \\
\hline NI & 150 & $7.5 \pm 1.5(1-22)$ & 99 & $6.7 \pm 4.7(1-25)$ & 152 & $6.8 \pm 6.5(1-20)$ \\
\hline NII & 138 & $8.1 \pm 1.7(1-19)$ & 80 & $6.8 \pm 6.2(1-22)$ & 91 & $6.9 \pm 6.1(1-21)$ \\
\hline NIII & 127 & $10.1 \pm 3.3(1-24)$ & 58 & $8.8 \pm 7.1(1-25)$ & 75 & $9.2 \pm 6.3(1-19)$ \\
\hline NIV & 119 & $13.8 \pm 2.6(1-31)$ & 49 & $8.5 \pm 6.7(1-34)$ & 58 & $7.7 \pm 6.2(1-33)$ \\
\hline NV & 109 & $13.6 \pm 3.5(1-44)$ & 41 & $8.6 \pm 7.2(1-35)$ & 51 & $8.4 \pm 6.7(1-42)$ \\
\hline Female & 53 & $14.5 \pm 2.7(1-50)$ & 22 & $9.1 \pm 7.6(1-46)$ & 25 & $1.3 \pm 1.5(1-46)$ \\
\hline Male & 37 & $12.7 \pm 4.2(1-53)$ & 19 & $9.9 \pm 8.7(1-44)$ & 23 & $6.6 \pm 9.1(1-32)$ \\
\hline
\end{tabular}

NI-NV: 1 st to 5 th instar nymphs. 


\section{DISCUSSION}

As in most previously studied Mexican triatomine species (Galvão et al. 1995, Martínez-Ibarra et al. 2001, 2003a,b, 2004, 2005, 2006), in the present study hatching rates were above $70 \%$ for all three species, with mean incubation periods of about 20 days. This reflects the fact that maintenance conditions were favorable for a proper development of these species. Hatching rates were slightly lower than those reported for two of the most important vectors of Chagas disease, Triatoma dimidiata Latreille and Triatoma infestans (Klug) (Zeledón et al. 1970a, Rabinovich 1972). In the present study, the egg hatching rate $(75 \%)$ for $T$. gerstaeckeri was similar to the rates reported for this species $(71.2 \%$ and $66.9 \%$ ) in two previous studies using specimens from Lackland Air Force Base, in Texas, and from General Terán, Nuevo León (Pippin 1970, Galavíz-Silva et al. 1991b). Both areas are near the locality where the parent specimens of our colony were collected. Egg hatching rate in $T$. protracta $(76 \%)$ was similar to the rates in two related former subspecies of the T. protracta complex (now sinonimized to $T$. protracta): T. $p$. navajoensis Ryckman (74.2\%) and T. p. zacatecensis Ryckman (81.9\%), both fed on rabbits (Ryckman 1962).

Mean egg-to-adult development time in $T$. gerstaeckeri was longer than in most related species of the Meccus phyllosomus complex (Martínez-Ibarra et al. 2003a,b, 2004, 2005, 2006). It was also longer than development time in T. gerstaeckeri from Texas (Pippin 1970), but similar to the mean development time recorded using a Mexican population of the same species, from an area close to that of the former study (Galavíz-Silva et al. 1991b). Longer development times in Mexican rather than Texan populations of $T$. gerstaeckeri may be due to the use of rabbits as blood meal sources, as in the present study and in Galavíz-Silva et al. (1991b), whereas Texan T. gerstaeckeri was fed on Neotoma micropus micropus by Pippin (1970), a host more frequently associated with $T$. gerstaeckeri than rabbits.

Triatoma protracta had a mean egg-to-adult development time of about eight months in the present study, almost twice as long as the time (129 days in females and 136.8 in males) recorded for the former T. $p$. navajoensis (sinonimized to $T$. protracta), fed on rabbits (Ryckman 1962). On the other hand, T. lecticularia had the shortest development time of all three species in the present study, apparently because its specimens had a higher degree of adaptation to laboratory conditions. This is similar to the reported behavior in Meccus mazzottii (Usinger) and M. phyllosomus (Burmeister) concerning M. bassolsae (Alejandre-Aguilar et al.) on the cohorts fed on hens as well as in the cohorts fed on rabbits (Martínez-Ibarra et al. 2006). The mean development time of T. lecticularia in the present study was also shorter than the time reported for specimens of this species that had grown in Brazil at a temperature of $28 \pm$ $0.1^{\circ} \mathrm{C}$ and $70 \pm 5 \% \mathrm{RH}$ (318.1 and 317 days in males and females, respectively) (Silva et al. 1993). The mean eggto-adult development time of the three studied species were similar to the time (240 days; range 180-336 days) reported for $T$. dimidiata, one of the most important vectors, which was fed fortnightly on rabbits and maintained at $26.5 \pm 0.5^{\circ} \mathrm{C}$ and $50 \pm 5 \% \mathrm{RH}$ (Zeledón et al. 1970b), and longer than the time (141 days) reported for specimens of $T$. infestans, another very important vector of Chagas disease, which was fed weekly on hens and maintained at $26 \pm 1{ }^{\circ} \mathrm{C}$ and $60 \pm 10 \% \mathrm{RH}$ (Rabinovich 1972). Drier conditions (30\% RH), which are closer to the natural conditions of the places where T. lecticularia is usually collected, probably favored their faster development in the current study.

On average, $70-80 \%$ of most instars of the three species studied here required three meals to molt to the next instar. Mean number of blood meals necessary for molting to the next instar was similar in all three species. It was also similar to some species values related to T. gerstaeckeri, such as Meccus longipennis (Usinger) in both cohorts (fed on hens or on white rats), Meccus pallidipennis (Stål) (fed on rabbits) and Triatoma barberi (Usinger) (fed on rabbits); however, it was smaller than the number of meals in Triatoma nitida (Usinger) (fed on pigeons); the two latter being related to T. lecticularia and T. protracta (Galvão et al. 1995, Martínez-Ibarra et al. 2004, 2005, Martínez-Ibarra \& Novelo-López 2004).

Mortality rates were lower in $T$. gerstaeckeri than in the other two species studied, as in the case of certain related species, such as $T$. dimidiata (fed on rabbits) and M. longipennis (Martínez-Ibarra et al. 2001, 2004). On the other hand, mortality indexes for T. lecticularia and T. protracta were similar to that of larger Mexican species, such as M. picturatus (Usinger) (fed on rabbits or on hens) and M. bassolsae (fed on rabbits) (MartínezIbarra et al. 2003b, 2006). Whereas, the indexes were higher than those for T. barberi $(25.5 \%)$ and $T$. nitida $(57.6 \%)-$ two species related to T. lecticularia and $T$. protracta - and higher than those for T. rubida (Uhler) (34.6\%; fed on rabbits), a species similar in size to $T$. lecticularia, which was collected in the same geographic area of T. protracta (Galvão et al. 1995, Martínez-Ibarra et al. 2005).

As for other species related to T. gerstaeckeri (those of the M. phyllosomus complex and T. dimidiata), to $T$. lecticularia and to T. protracta (T. barberi and T. nitida), the instar mortality rate for three species herein analyses showed an irregular pattern. The highest mortality rates in all three species were found in first or fifth instar nymphs, as recorded for numerous other species of Mexican Triatominae (Galvão et al. 1995, MartínezIbarra et al. 2001, 2003a,b, 2005, 2006). As in these latter species, in our study, youngest nymphs' mortality was apparently due to insects feeding incapacity, since usually no significant intestinal content was found in dead specimens (analyzed through direct observation). On the other hand, mortality in older nymphs seemed to occur in the molting period.

Mean FT was significantly longer $(p<0.05)$ than 10 min for most instars of T. gerstaeckeri. This is similar to the FT reported for this species, from Texas, and for Mexican specimens from an area near the locality where the parent specimens of our study were collected (Pippin 1970, Galavíz-Silva et al. 1991b). Mean FT for $T$. 
lecticularia were similar to the times reported for $T$. barberi, a related species (Martínez-Ibarra et al. 2005). Like several other parameters in this study, mean FT in all three species is well within the range of published data for some other related Mexican species, such as $T$. dimidiata, the species of $M$. phyllosomus complex, as well as T. barberi and T. rubida (Martínez-Ibarra et al. 2001, 2003a,b, 2004, 2005, 2006), and the three most important vectors of Chagas disease, like $T$. dimidiata, T. infestans and Rhodnius prolixus Stål (all these three fed on mice) (Zeledón et al. 1977).

Postfeeding defecation delay was less than $10 \mathrm{~min}$ for younger nymphs of $T$. gerstaeckeri, all instars of $T$. lecticularia, as previously reported for the last species (Nogueda-Torres et al. 2000), and for all instars of $T$. protracta. The hypothesis presented by Dias (1956) and, later on, supported by Zeledón et al. (1977), points out that triatomines that defecate before 10 min postfeeding are potentially effective transmitters of $T$. cruzi. Therefore, all T. lecticularia and T. protracta instars may be potentially effective vectors of $T$. cruzi. From this point of view, these two Triatoma species may be considered as potentially effective in transmitting $T$. cruzi as $T$. infestans and Rh. prolixus (Zeledón et al. 1977).

It follows from Zeledón et al. (1977) that first and second-instar nymphs of $T$. gerstaeckeri may be important because of their capacity to transmit T. cruzi, as is also the case of specimens of this species from a nearby area in Mexico (Galavíz-Silva et al. 1991b). Unfortunately, comparison with Texan specimens is not possible, since the author of the latter study reported the numbers and percentages of triatomines defecating $2 \mathrm{~min}$ after feeding, but omitted data on the rest of the specimens of the study (Pippin 1970). Triatoma gerstaeckeri took longer to defecate than most related species (particularly those of $M$. phyllosomus complex), except for $M$. bassolsae, which presented similar times, and $T$. dimidiata and T. nitida, both with longer defecation delays (Galvão et al. 1995, Martínez-Ibarra et al. 2001, 2003a,b, 2004, 2006).

Most parameters used in this study lead us to conclude that all three species may be major potential vectors of $T$. cruzi for human populations in Mexican areas where these species are currently found, as previously suggested by several field studies (Martínez-Ibarra et al. 1992, Beard et al. 2003, Galvão et al. 2003, MolinaGarza et al. 2006, Hanford et al. 2007).

\section{ACKNOWLEDGMENTS}

To Verónica Ventura for helping in carrying out this study.

\section{REFERENCES}

Beard CB, Pye G, Steurer FJ, Rodríguez R, Campean R, Peterson AT, Ramsey J, Wirtz RA, Robinson LE 2003. Chagas disease in a domestic transmission cycle in Southern Texas, USA. Emerg Inf Dis 9: 103-105.

Cruz-Reyes A, Pickeri-López JM 2006. Chagas disease in Mexico: an analysis of geographical distribution during the past 76 yearsA review. Mem Inst Oswaldo Cruz 101: 34-354.

Dias E 1956. Observação sobre eliminação de dejeções e tempo de sucção em alguns triatomíneos sulamericanos. Mem Inst Oswaldo Cruz 54: 115-124.
Galavíz-Silva L, Arredondo-Cantú JM, Vilma-Ramírez H 1991a. House Triatominae at the San Juan de Vaquerias communal land ("Ejido") in Nuevo Leon, Mexico. Bord Health 7: 16-22.

Galavíz-Silva L, Jiménez-Guzmán F, Fernández-Salas I, MolinaGarza ZJ, Martínez-Ibarra JA 1991b. Datos biológicos de Triatoma gerstaeckeri Stål bajo condiciones de laboratorio. Pub Biol FCB/UANL, México 5: 81-87.

Galvão C, Carcavallo R, Rocha-Da Silva D, Jurberg J 2003. A checklist of the current valid species of the subfamily Triatiominae Jeannel, 1919 (Hemiptera: Reduviidae) and their geographical distribution, with nomenclatural and taxonomic notes. Zootaxa 202: 1-36.

Galvão C, Jurberg J, Cunha V, Pinto de Mello R 1995. Biologia do Triatoma nitida Usinger, 1939 em laboratório (Hemiptera: Reduviidae). Mem Inst Oswaldo Cruz 90: 657-663.

García-Pérez JA, Blanco-Piñón A, Navarro-Fernández E 1997. Comportamiento sexual de Triatoma gerstaeckeri Stål (Hemiptera: Reduviidae). Acta Zool Mex 70: 55-63.

Hanford EJ, Zhan FB, Lu Y, Giordano A 2007. Chagas disease in Texas: Realizing the significance and implications of evidence in the literature. Soc Sc Med 65: 80-94.

Jurberg J, Costa JM 1989. Estudos sobre a resistência ao jejum e aspectos nuticionais de Triatoma lecticularia (Stål,1859) (Hemiptera, Reduviidae, Triatominae). Mem Inst Oswaldo Cruz 84: 393-399.

Licón-Trillo A 2006. Infección de Triatoma recurva por Trypanosoma cruzi en un campamento minero de Urique, Chihuahua (México). Rev Fac Sal Púb Nut 7: 1-8.

Martínez-Ibarra JA, Alejandre-Aguilar R, Torres-Morales A, Trujillo-García JC, Nogueda-Torres B, Trujillo-Contreras F 2006. Biology of three species of the Meccus phyllosomus complex (Hemiptera: Reduviidae Triatominae) fed on blood of hens and rabbits. Mem Inst Oswaldo Cruz 101: 787-794.

Martínez-Ibarra JA, Galavíz-Silva L, Lara-Campos C, TrujilloGarcía JC 1992. Distribución de los triatominos asociados al domicilio humano en el municipio de General Terán, Nuevo León, México. Southwest Entomol 17: 261-266.

Martínez-Ibarra JA, Grant-Guillén Y, Martínez-Grant DM 2003a. Feeding, defecation and development times of Meccus longipennis Usinger 1939 (Hemiptera: Reduviidae: Triatominae) under laboratory conditions. Mem Inst Oswaldo Cruz 98: 899-903.

Martínez-Ibarra JA, Grant-Guillén Y, Nogueda-Torres B, TrujilloContreras F 2004. Influence of the blood meal source on the biology of Meccus longipennis Usinger 1939 (Hemiptera: Reduviidae) under laboratory conditions. J Am Mosq Control Assoc 20:328-330.

Martínez-Ibarra JA, Miguel-Álvarez A, Arredondo-Jiménez JI, Rodríguez-López MH 2001. Update on the biology of Triatoma dimidiata Latreille (Hemiptera: Reduviidae) under laboratory conditions. J Am Mosq Control Assoc 17: 209-210.

Martínez-Ibarra JA, Nogueda-Torres B, Paredes-González E, Alejandre-Aguilar R, Solorio-Cibrián M, Barreto SP, GómezEstrada HI, Trujillo-García JC 2005. Development of Triatoma rubida sonoriana, Triatoma barberi, and Meccus mazzottii (Heteroptera, Reduviidae) under laboratory conditions. J Am Mosq Control Assoc 21: 310-315.

Martínez-Ibarra JA, Novelo-López M 2004. Biological parameters 
of Triatoma pallidipennis Stål (Hemiptera: Reduviidae) under laboratory conditions. Folia Entomol Mex 43: 313319.

Martínez-Ibarra JA, Novelo-López M, Hernández-Robles MR, Grant-Guillén Y 2003b. Influence of the blood meal source on the biology of Meccus picturatus Usinger 1939 (Hemiptera: Reduviidae: Triatominae) under laboratory conditions. Mem Inst Oswaldo Cruz 98: 227-232.

Molina-Garza ZJ, Galavíz-Silva L, Rosales-Encinas JL, MolinaGarza DP 2006. Detección de Trypanosoma cruzi (Protozoa, Kinetoplastida) en el estado de Nuevo León, México. Rev Sal Púb Nut 11: 34-37.

Molina-Garza ZJ, Rosales-Encinas JL, Galavíz-Silva L, MolinaGarza DP 2007. Prevalencia de Trypanosoma cruzi en triatominos silvestres de Nuevo León, México. Sal Púb Méx 49: $37-44$

Navin TR, Roberto RR, Juranek DD, Limpakarnjarat K, Mortenson EW, Clover JR, Yescott RE, Taclindo C, Steurer F, Allain D 1985. Human and sylvatic Trypanosoma cruzi infection in California. Am J Public Health 75: 366-369.

Nogueda-Torres B, Alejandre-Aguilar R, Isita-Tornell L, Camacho AD 2000. Defaecation pattern in seven species of triatomines (Insecta, Reduviidae) present in Mexico. Rev Lat Microbiol 42: $145-148$.

Paredes EA, Valdez-Miranda J, Nogueda-Torres B, AlejandreAguilar R, Canett-Romero R 2001. Vectorial importance of triatomine bugs (Hemiptera: Reduviidae) in Guaymas, Mexico. Rev Lat Microbiol 43: 119-122.

Pippin WE 1970. The biology and vector capability of Triatoma sanguisuga texana Usinger and Triatoma gerstaeckeri (Stål) compared with Rhodnius prolixus (Stål) (Hemiptera: Triatominae). J Med Entomol 7: 30-45.
Rabinovich J 1972. Vital statistics of triatominae (Hemiptera: Reduviidae) under laboratory conditions. I Triatoma infestans Klug. J Med Entomol 9: 351-370.

Ryckman R 1962. Biosystematics and hosts of the Triatoma protracta complex in North America. Univ California Publ Ent 27: 93-239.

Seigler DV, Lampman RL 2000. Isobutyric acid from the Brindley's glands of Triatoma lecticularia. J Am Mosq Control Assoc 16: 36-37.

Silva IG, Santos LG, Nakano R 1993. Ciclo evolutivo de Triatoma lecticularia (Stål, 1859) (Hemiptera, Reduviidae). Rev Patol Trop 22: 259-263.

Torres-Estrada JL, Martínez-Ibarra JA, García-Pérez JA 1993. Sitios de reposo intradomiciliarios de ninfas de quinto estadio de Triatoma gerstaeckeri Stål en laboratorio. Southwest Entomol 18: 45-49.

Torres-Estrada JL, Martínez-Ibarra JA, García-Pérez JA 2002. Selection of resting sites of Triatoma gerstaeckeri (Stål) (Hemiptera: Reduviidae) females under laboratory and field conditions. Folia Entomol Mex 41: 63-66.

Zárate LG, Zárate RJ 1985. A checklist of the Triatominae (Hemiptera: Reduviidae) of Mexico. Int J Entomol 27: 102-127.

Zeledón R, Alvarado R, Jirón JF 1977. Observations on the feeding and defecation patterns of three triatomine species (Hemiptera: Reduviidae). Acta Trop 34: 65-77.

Zeledón R, Guardia VM, Zúñiga A, Swartzwelder JC 1970a. Biology and ethology of Triatoma dimidiata (Latreille, 1811). II Life span of adults and fecundity and fertility of females. $J$ Med Entomol 7: 462-469.

Zeledón R, Guardia VM, Zúñiga A, Swartzwelder JC 1970b. Biology and ethology of Triatoma dimidiata (Latreille, 1811). I Life cycle, amount of blood ingested, resistance to starvation, and size of adults. J Med Entomol 7: 313-319. 